\title{
Generation of 3D Surface Models from Scanning Electron Microscope Images
}

\author{
Timothy Amish ${ }^{1}$, Bryan T. Hansen ${ }^{1}$, and Elizabeth R. Fischer ${ }^{1}$
}

${ }^{1}$ Electron Microscopy Unit, Research Technologies Branch, Rocky Mountain Laboratories, National Institute of Allergy and Infectious Diseases, NIH Hamilton, MT 59840

The study of host-pathogen interactions begins with the attachment of a bacteria or virus to a host cell. Studying this initial interaction which often involves significant cytoskeletal rearrangements and membrane changes can provide clues for entry and egress mechanisms. Scanning electron microscopy (SEM) provides a widely used tool to qualitatively determine surface changes that occur but is limited quantitatively as the resulting image is still a twodimensional output. Optimizing an image capture strategy and utilizing Autodesk's Memento Software, allowed 3-dimensional topographic reconstructions from SEM images. [Ref. 1]

New software is becoming increasingly sophisticated and capable of accurate qualitative and quantitative analyses. Autodesk has created programs that utilize images from multiple orientations to create a 3D model for a variety of applications: 3D printing, medical, industrial, and media. A user inputs a grouping of images at different tilts and rotations around an object. The Autodesk programs are able to pick out similar points in the images and compare them which results in correlating the location of photos relative to each other in 3D space. However, these $2 \mathrm{D}$ to $3 \mathrm{D}$ programs have inherent limitations. The programs cannot reliably generate volumes from objects with little surface texture or symmetry as it may misplace points during correlation. The more unique and complex features are the more likely they will stitch correctly. These programs were primarily designed to collect images by physically moving a camera around a fixed point. By contrast we wanted to develop a data collection scheme in an SEM where the camera position is fixed and the specimen is either physically or raster rotated, tilted or combinations thereof relative to the fixed secondary electron detector.

Many trials were performed with variables that included specimen variability, magnification, and number of images, capture settings, tilts and rotations to optimize the resulting models. Multiple tilts with little rotation or fixed tilts with only physical rotation showed little promise with many large gaps and distortions within the model using Autodesk's 123D Catch program. (FIG. 1, Ref. 2) In contrast, the best results were achieved by rotation around a fixed angle by physically tilting the sample to plus 45 degrees relative to the detector. (FIG 2 A \& B) Moving the stage in a positive direction in 4 degree increments and countering with a negative 4 degree raster rotation through 360 degrees around the sample gave the most complete model generation using Autodesk's Memento software. (FIG 3) Once the positions of the images were determined by cross-correlation, points were arranged and projected into 3D space. The created points were then used to produce thousands of interconnected triangles which provides the model with depth and contours. The volumes can be visualized with a variety of surface textures. (FIG 4 A-C)

The resulting 3D models can be useful for quantitative analysis of surface structures. Complex measurements can be made with ease. A qualitative understanding of specimens can be observed from a computed environment or by 3D printing the resulting 3D model. Typically it takes a day to generate images and create a model without expensive software or hardware, making the model generation easily accessible with a standard SEM. 


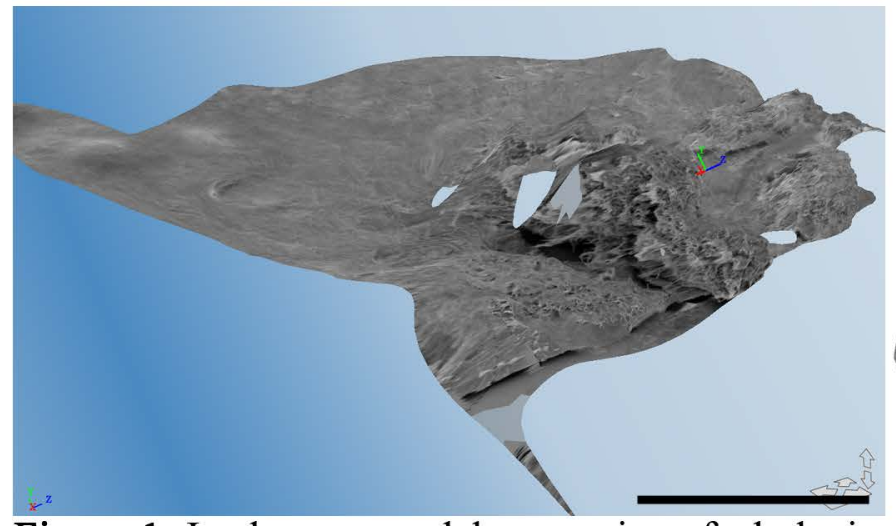

Figure 1. Inadequate model generation of ebolavirus infected Vero cell from data collection at a 45 degree stage tilt and 360 degrees of rotation by 8 degree increments. Scale bar $=10 \mathrm{um}$

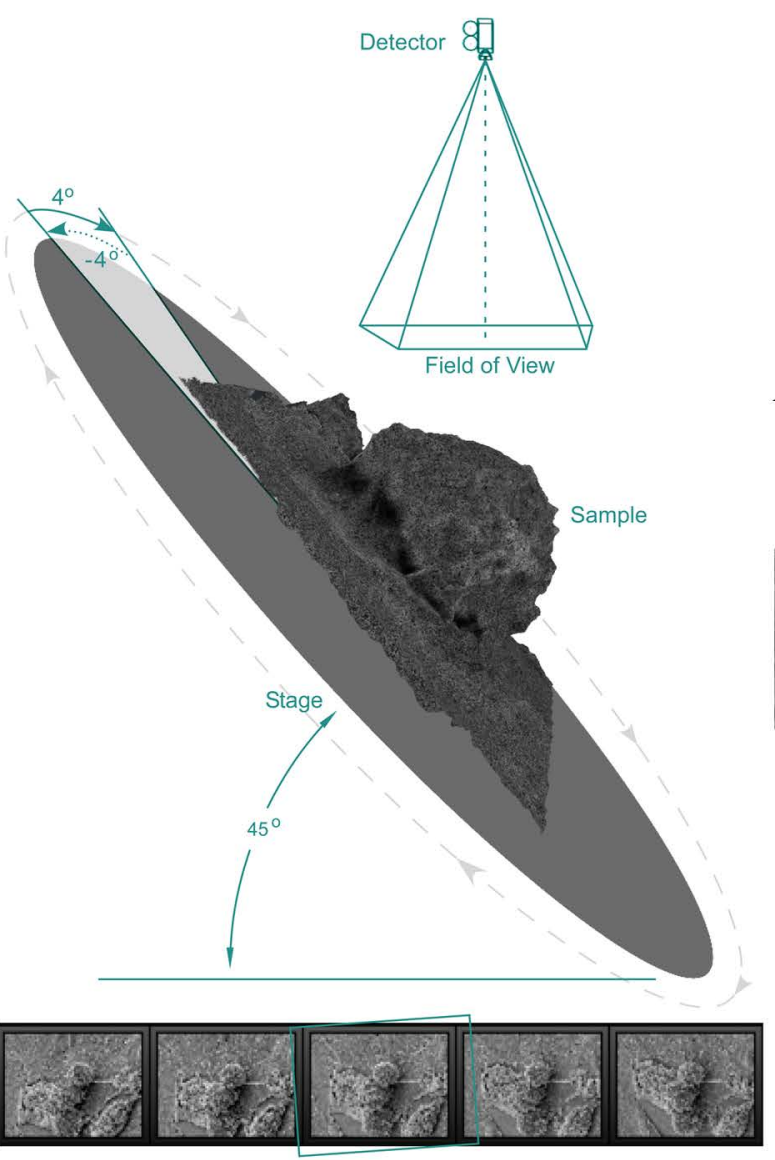

Figure 3. Schematic of the the optimal image collection scheme used to generate model in Fig. 2

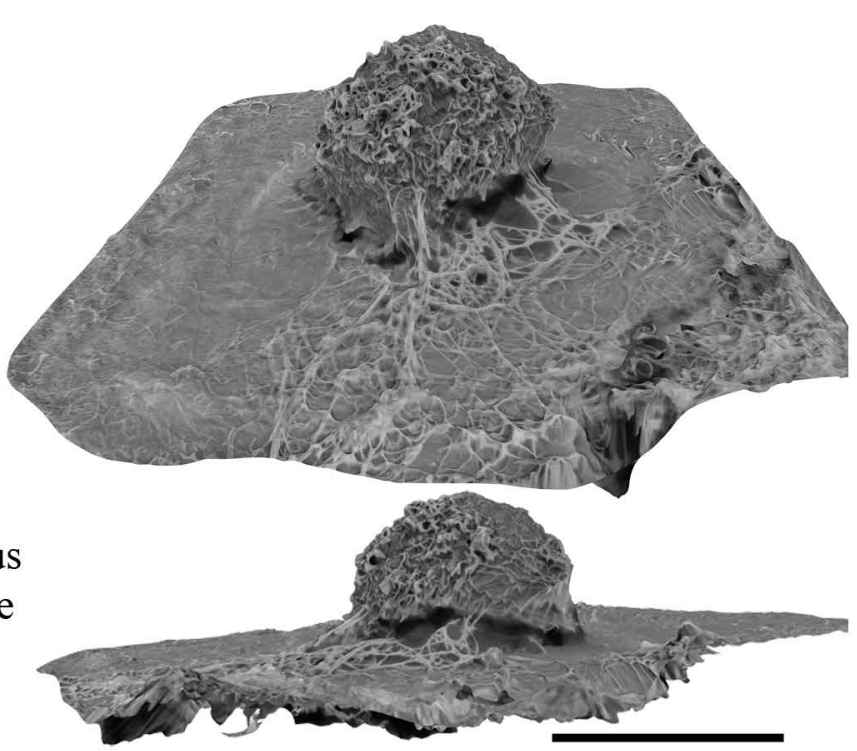

Figure 2. Two orientations of improved model generation of the same specimen with images collected at a 45 degree stage tilt and 360 degrees of physical rotation by 4 degree increments and equivalent negative degree raster rotations back. Scale bar $=10$ um.

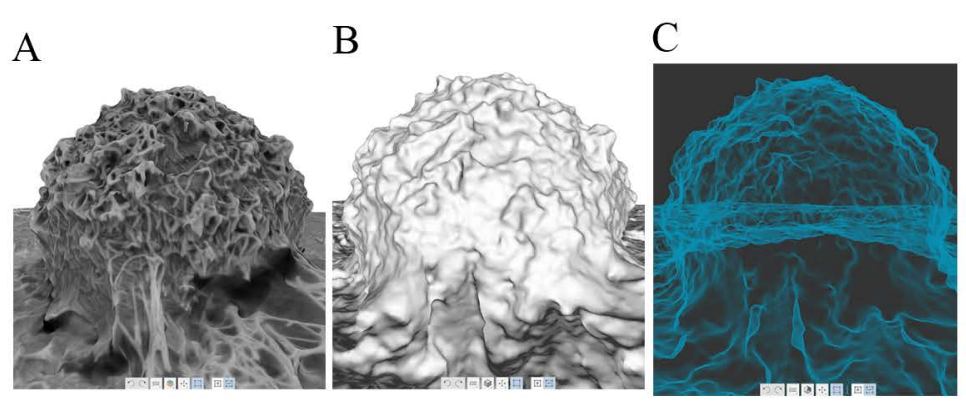

Figure 4 A-C. The same model showing different rendering styles:A is "Textured," B is "Solid" and, $\mathrm{C}$ is " $\mathrm{X}$-ray"

The authors would like to acknowledge the RML NIAID, NIH Electron Microscopy group for helpful discussions and Austin Athman for graphic arts assistance. This work was supported by NIAID intramural research program 